\title{
Relationship between serum MMP-9 level and prognosis after radical resection for Hilar cholangiocarcinoma patients ${ }^{1}$
}

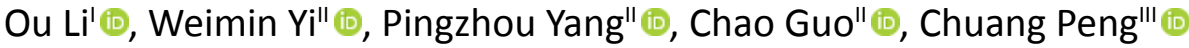

'MD, Department of Hepatobiliary Surgery, Hunan Provincial People's Hospital, People's Republic of China. Technical procedures, manuscript writing.

"MD, Department of Hepatobiliary Surgery, Hunan Provincial People's Hospital, People's Republic of China. Technical procedures, analysis of data.

I'MD, Department of Hepatobiliary Surgery, Hunan Provincial People's Hospital, People's Republic of China. Design of the study, critical revision.

\begin{abstract}
Purpose: To analyze the preoperative serum matrix metalloproteinase-9 (MMP-9) levels and prognosis of patients with hilar cholangiocarcinoma $(\mathrm{HC})$ undergoing radical resection.

Methods: Preoperative serum MMP-9 levels in patients with $\mathrm{HC}$ undergoing radical resection were detected by enzyme-linked immunosorbent assay (ELISA). The ROC curve assay was used to analyze the preoperative serum MMP-9 level to determine the most valuable cutoff point. The relationship between MMP-9 and clinicopathological features of HC patients was analyzed. Kaplan-Meier method was used to analyze the prognostic factors, and COX regression model was used to analyze the independent risk factors affecting prognosis.

Results: Preoperative serum MMP-9 levels were significantly elevated in the death patients compared with the survival patients. The most valuable cut-off point for preoperative serum MMP-9 for prognosis was $201.93 \mathrm{ng} / \mathrm{mL}$. Preoperative serum MMP-9 was associated with Bismuth-Corlette classification) and lymph node metastasis. Kaplan-Meier analysis showed that MMP-9, Bismuth-Corlette classification, Lymph node metastasis, Portal vein invasion, Hepatic artery invasion, Liver invasion, Incised margin, and Preoperative biliary drainage were related to prognosis. Cox regression model confirmed that hepatic artery invasion, liver invasion, incised margin, and MMP-9 have the potential to independence predicate prognosis in $\mathrm{HC}$ patients.
\end{abstract}

Conclusion: Preoperative serum MMP-9 has high predictive value for prognosis and is an independent influencing factor for the prognosis of patients with hilar cholangiocarcinoma.

Key words: Klatskin Tumor. Matrix Metalloproteinase 9. Postoperative Period. Prognosis. 


\section{Introduction}

Hilar cholangiocarcinoma (HC), also known as Klatskin tumor, is a malignant tumor that grows in the confluence of the left and right hepatic ducts and the common hepatic duct. In cholangiocarcinoma, HC is more common that is accounting for about 58\%$66 \%^{1}$. HC has a high mortality rate in the early stages and is considered to be one of the most deadly malignant tumors ${ }^{2}$. However, the cause of $\mathrm{HC}$ is not yet clear. Liver resection and liver transplantation in HC patients are currently the only radical surgery ${ }^{3}$, however, only a small number of patients still have the opportunity to undergo radical surgery after diagnosis, and the postoperative prognosis is poor. Other adjuvant therapies such as chemotherapy and radiotherapy have not achieved satisfactory results in improving the prognosis of patients ${ }^{4}$. Studying factors related to prognosis after surgical treatment and finding related markers for preventing $\mathrm{HC}$ recurrence or metastasis are of great significance for improving the longterm survival of $\mathrm{HC}$ patients.

Matrix metalloproteinase-9 (MMP9), also known as gelatinase $B$ or type IV collagenase $B$, is a zinc-dependent proteolytic enzyme involved in the degradation of many different components of the basement membrane and subsequent remodeling of extracellular matrix (ECM) $)^{5}$. MMP-9 not only plays a key role in the development of cancers, but also their characteristics of being secreted into the blood stream have inspired many researchers to evaluate the associations between circulating level of MMP9 and clinicopathological characteristics of cancers ${ }^{6}$. Clinical studies have shown that high expression of MMP-9 is associated with clinical pathological features of cancer such as lymph node metastasis and tumor differentiation?. Elevated serum MMP-9 often suggests a poor prognosis ${ }^{8}$. However, there are few studies on the relationship between MMP-9 and the histopathological features and prognosis of $\mathrm{HC}$. There is currently no correlation report on the preoperative serum MMP-9 level predicting the prognosis of $\mathrm{HC}$.

In this study, patients with $\mathrm{HC}$ radical surgery were enrolled. Enzyme-linked immunosorbent assay (ELISA) was used to analyze the most valuable cut-off point of preoperative serum MMP-9 for the prognosis of $\mathrm{HC}$ radical resection, and to explore the influencing factors of prognosis of $\mathrm{HC}$ radical mastectomy. We hope to provide appropriate targets for prognosis monitoring after $\mathrm{HC}$.

\section{Methods}

This study was approved by the Ethics Committee of the Hunan Provincial People's Hospital.

Two hundred and forty-one patients with HC who were admitted to Hunan Provincial People's Hospital from March 2010 to March 2013 were included in the study. All patients underwent radical surgery and were diagnosed by pathology after surgery. Patients with perioperative death were excluded. Pathological results were excluded from mixed liver cancer, as well as patients who were lost to follow-up. The causes of death in follow-up cases were surgical factors (non-other cases, such as heart disease). Finally, 181 eligible cases were included, including 103 males and 78 females, aged $35-78$ years. All patients were informed of the relevant conditions of the study and the required indicators to be recorded before surgery. Patients were signed informed consent and followed up regularly. The deadline for follow-up is June 30, 2018. The overall survival (OS) time is from the date of surgery to the date of death or the date of 
follow-up. All experiments were performed at Department of Hepatobiliary Surgery, Hunan Provincial People's Hospital, China. Patient enrollment and follow-up are shown in Figure 1.

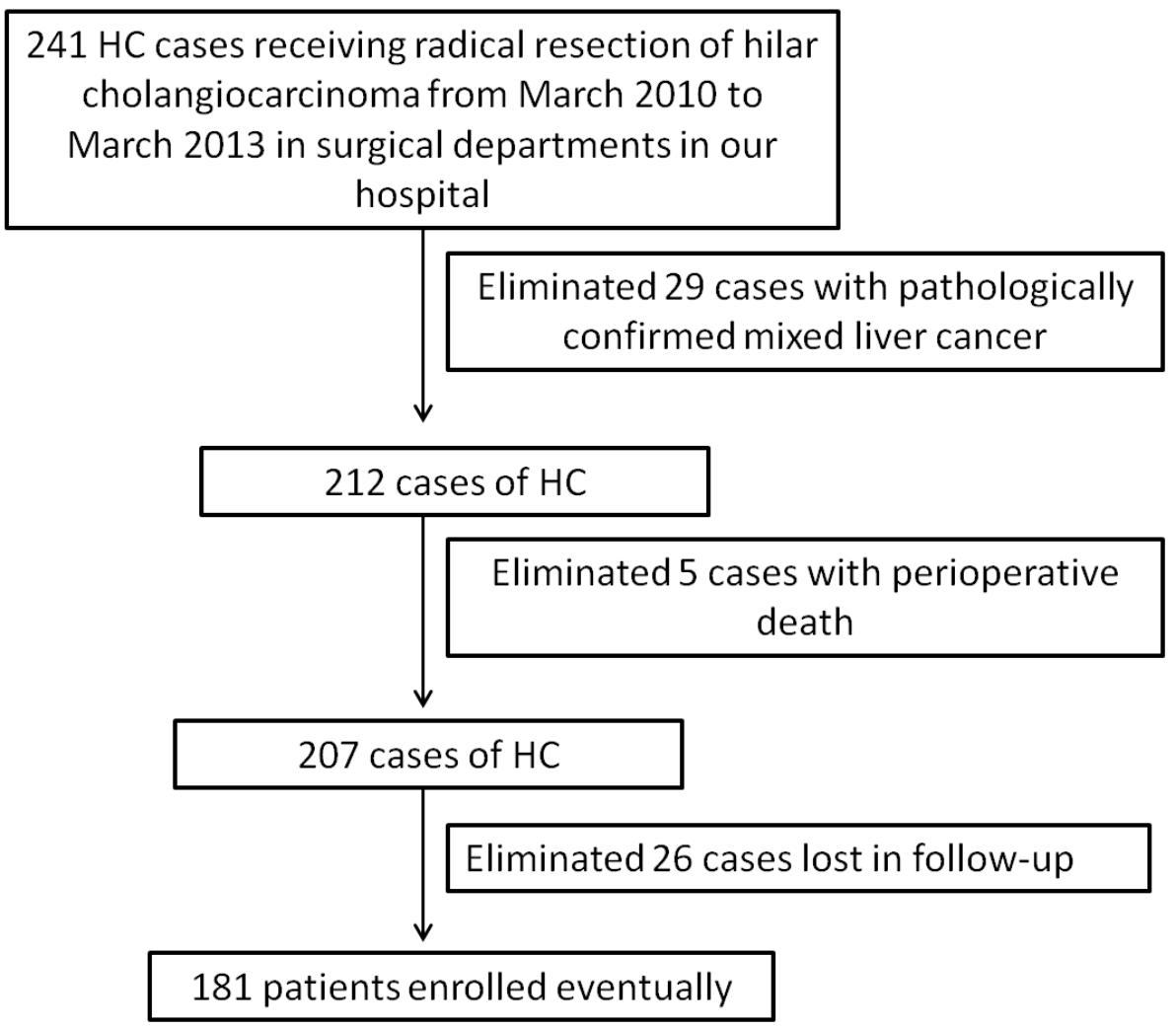

Figure 1 - Inclusion criteria and censored data of the patients.

\section{Detection of serum MMP-9 content}

Three milliliters of venous peripheral blood of HC patients was collected before radical surgery, and centrifuged to obtain serum, which was then stored at $-80^{\circ} \mathrm{C}$. The concentration of MMP-9 was measured by ELISA. ELISA kit for MMP-9 WAS purchased from R\&D Systems Inc. Assays were performed according to the manufacturer's instructions.

\section{Statistical analyses}

Analysis was performed using SPSS 17.0 software (SPSS Inc, Chicago,
IL). Measurement data conforming to the normal distribution were expressed as mean \pm standard deviation(mean $\pm S D$ ), Student $t$ test was used for comparing the average of two groups. The predictive efficacy of serum MMP-9 on prognosis was assessed using the receiver operating characteristic curve (ROC). The most prognostic value of serum MMP-9 was calculated using the most approximate index. Count data was expressed in terms of number of cases (n), using $\chi 2$ test or Fisher exact probability method to analyze. The survival curve was drawn using the KaplanMeier method. Differences in survival curves 
were detected by Log-rank method. Cox regression model was established, and the significance level of the variables retained by the model: the entry criterion was $p<0.05$. At the $\alpha=0.05$ test level, the Backward LR method was used to screen out the independent risk factors affecting the prognosis of $\mathrm{HC}$ patients. The degree of influence of risk factors is expressed as the hazard ratio (HR). If $p<0.05$ then the difference was considered statistically significant.

\section{Results}

\section{Follow-up}

To the follow-up deadline, 120 of the 181 patients died, with a minimum survival time of 3 months, a maximum of 59 months, and a median survival of 46 months. The overall survival curve of the patient is shown in Figure 2. The 1-, 3-, and 5-year survival rates were $88.4 \%$ (160/181), 73.5\% (133/181), and $33.7 \%(61 / 181)$, respectively.

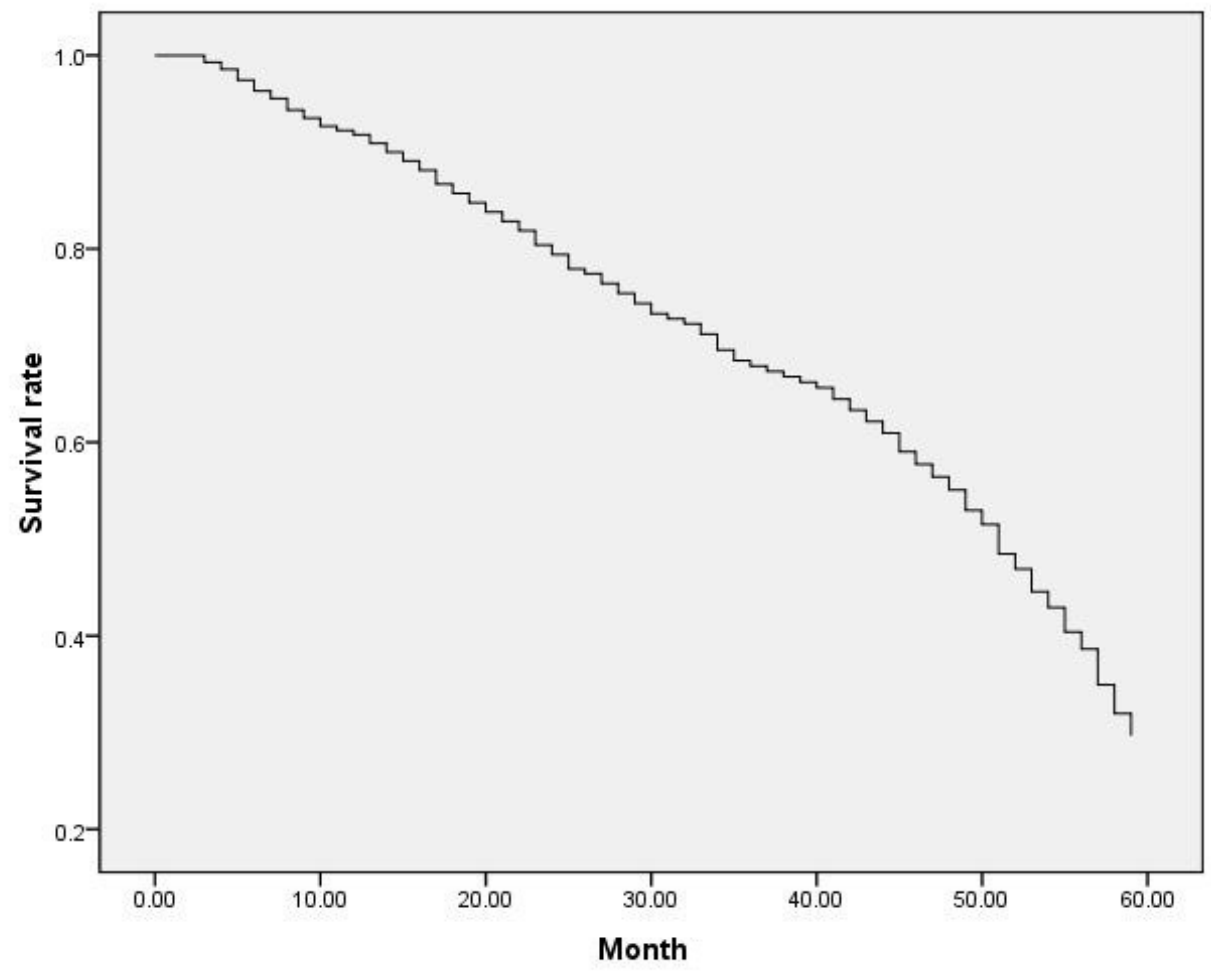

Figure 2 - Overall survival curve of HC patients after surgery.

The relationship between preoperative serum MMP-9 level and prognosis of HC patients

The preoperative serum MMP-9 concentration in 181 patients with $\mathrm{HC}$ was (216.7 \pm 72.1$) \mathrm{ng} / \mathrm{mL}$. Preoperative serum MMP-9 levels were significantly elevated in the death group compared with the survival group (155.50 \pm 41.79 vs. $251.57 \pm 62.02, p<0.001)$. To determine the most valuable MMP-9 level cutoff point for prognosis, we applied the ROC curve method for analysis. We found that the area under the ROC curve of serum MMP-9 levels predicting patient mortality was 0.896 (Fig. 3), 
confirming that preoperative serum MMP-9 has a higher value in predicting postoperative prognosis in $\mathrm{HC}$ patients. When the MMP-9 level was $201.93 \mathrm{ng} / \mathrm{mL}$, the Youden index was the largest. The sensitivity of MMP-9 $\geq 201.93$ $\mathrm{ng} / \mathrm{mL}$ for predicting death in $\mathrm{HC}$ patients was $80.33 \%$, and the specificity was $86.89 \%$. The result hinted that preoperative serum MMP-9 levels may be potential markers for predicting the prognosis of $\mathrm{HC}$ patients.

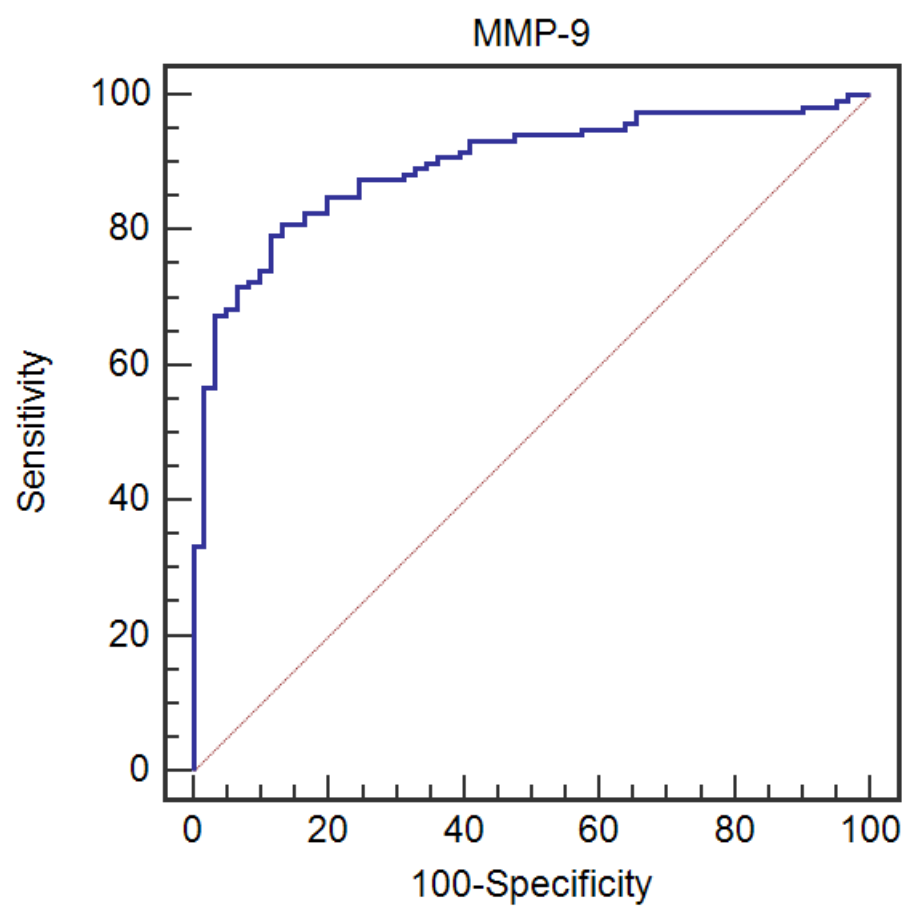

Figure 3 - Preoperative serum MMP-9 predicts ROC curve analysis of postoperative death in HC patients.

Relationship between serum MMP-9 level and clinicopathological features of HC patients

To analyze the relationship between preoperative serum MMP-9 and clinicopathological features of $\mathrm{HC}$ patients, we classified the variables related to clinicopathological features according to their own characteristics. As shown in Table
1, preoperative serum MMP-9 levels were not associated with age, sex, tumor size, differentiation, nerve invasion, portal vein invasion, hepatic artery invasion, liver invasion, incised margin, total bilirubin, preoperative biliary drainage and postoperativeradiotherapy, but were associated with Bismuth-Corlette classification ( $\chi 2=9.442, p=0.002)$ and lymph node metastasis $(\chi 2=9.026, p=0.003)$. 
Table 1 - Relationship between serum MMP-9 level and clinicopathological features of HC patients (n, \%).

\begin{tabular}{|c|c|c|c|c|c|}
\hline \multirow{2}{*}{ Item } & & \multirow[b]{2}{*}{$\mathbf{n}$} & \multicolumn{2}{|c|}{ MMP-9 (ng/mL) } & \multirow[b]{2}{*}{$\mathbf{p}$} \\
\hline & & & $\begin{array}{l}<201.93 \\
(n=75)\end{array}$ & $\begin{array}{l}\geq 201.93 \\
(n=106)\end{array}$ & \\
\hline \multirow{2}{*}{ Age } & $<60$ years & 97 & $38(50.7 \%)$ & $59(55.7 \%)$ & \multirow{2}{*}{0.507} \\
\hline & $\geq 60$ years & 84 & $37(49.3 \%)$ & $47(44.3 \%)$ & \\
\hline \multirow{2}{*}{ Sex } & Male & 103 & $42(56.0 \%)$ & $61(57.6 \%)$ & \multirow{2}{*}{0.836} \\
\hline & Female & 78 & $33(44.0 \%)$ & $45(42.4 \%)$ & \\
\hline \multirow{2}{*}{ Tumor size } & $<2.5 \mathrm{~cm}$ & 113 & $51(68.0 \%)$ & $62(58.5 \%)$ & \multirow{2}{*}{0.193} \\
\hline & $\geq 2.5 \mathrm{~cm}$ & 68 & $24(32.0 \%)$ & $44(41.5 \%)$ & \\
\hline \multirow{2}{*}{ Differentiation } & High and moderate & 174 & $72(96.0 \%)$ & 102(96.2\%) & \multirow{2}{*}{0.754} \\
\hline & Poor & 7 & $3(4.0 \%)$ & $4(3.8 \%)$ & \\
\hline \multirow{2}{*}{ Bismuth-Corlette classification } & $I+I I$ & 42 & $26(34.7 \%)$ & $16(15.1 \%)$ & \multirow{2}{*}{0.002} \\
\hline & $|I I a+I I| b+I V$ & 139 & $49(65.3 \%)$ & $90(84.9 \%)$ & \\
\hline \multirow{2}{*}{ Lymph node metastasis } & Negative & 117 & $58(77.3 \%)$ & $59(55.7 \%)$ & \multirow{2}{*}{0.003} \\
\hline & Positive & 64 & $17(22.7 \%)$ & $47(44.3 \%)$ & \\
\hline \multirow{2}{*}{ Nerve invasion } & Negative & 109 & $49(65.3 \%)$ & $60(56.6 \%)$ & \multirow{2}{*}{0.237} \\
\hline & Positive & 72 & $26(34.7 \%)$ & $46(43.4 \%)$ & \\
\hline \multirow{2}{*}{ Portal vein invasion } & Negative & 126 & $58(77.3 \%)$ & $68(64.2 \%)$ & \multirow{2}{*}{0.057} \\
\hline & Positive & 55 & $17(22.7 \%)$ & $38(35.8 \%)$ & \\
\hline \multirow{2}{*}{ Hepatic artery invasion } & Negative & 142 & $56(74.7 \%)$ & $86(81.1 \%)$ & \multirow{2}{*}{0.297} \\
\hline & Positive & 39 & $19(25.3 \%)$ & $20(18.9 \%)$ & \\
\hline \multirow{2}{*}{ Liver invasion } & Negative & 132 & $56(74.7 \%)$ & $76(71.7 \%)$ & \multirow{2}{*}{0.658} \\
\hline & Positive & 49 & $19(25.3 \%)$ & $30(28.3 \%)$ & \\
\hline \multirow{2}{*}{ Incised margin } & Negative(R0) & 151 & $66(88.0 \%)$ & $85(80.2 \%)$ & \multirow{2}{*}{0.164} \\
\hline & Positive(R1) & 30 & $9(12.0 \%)$ & $21(19.8 \%)$ & \\
\hline \multirow{2}{*}{ Total bilirubin } & $<100 \mathrm{mg} / \mathrm{L}$ & 77 & $35(46.7 \%)$ & $42(39.6 \%)$ & \multirow{2}{*}{0.345} \\
\hline & $\geq 100 \mathrm{mg} / \mathrm{L}$ & 104 & $40(53.3 \%)$ & $64(60.4 \%)$ & \\
\hline \multirow{2}{*}{ Preoperative biliary drainage } & Undone & 126 & $54(72.0 \%)$ & $72(67.9 \%)$ & \multirow{2}{*}{0.557} \\
\hline & Done & 55 & $21(28.0 \%)$ & $34(32.1 \%)$ & \\
\hline \multirow{2}{*}{ Postoperative radiotherapy } & Undone & 109 & $47(62.7 \%)$ & $62(54.5 \%)$ & 257 \\
\hline & Done & 72 & $28(37.3 \%)$ & $44(41.5 \%)$ & \\
\hline
\end{tabular}

High MMP-9 levels are correlated with poor prognosis in HC patients

To explore whether clinical pathological parameters and preoperative serum MMP9 levels were associated with OS, we utilized
Kaplan Meier survival analysis and log-rank test (Table 2, Fig. 4). The median survival time was 34.5 months in patients with high MMP-9 (MMP-9 $\geq 201.93$ ) and 50.9 months in patients with low MMP-9 (MMP-9<201.93 ng/mL). The 
Kaplan-Meier results showed (Table 2, Fig. 4H) that patients with high MMP-9 had a significantly lower median survival time than patients with low MMP-9 (log-rank $\chi 2=63.488, p<0.001$ ). Subsequently, Bismuth-Corlette classification (log-rank $\chi 2=4.393, p=0.036)$, Lymph node metastasis (log-rank $\chi 2=7.284, p=0.007)$, Portal vein invasion (log-rank $\chi 2=4.905, p=0.027$ ), Hepatic artery invasion (log-rank $\chi 2=5.481$, $p=0.019$ ), Liver invasion (log-rank $\chi 2=4.705$, $p=0.030$ ), Incised margin (log-rank $\chi 2=10.122$, $p=0.001)$, and Preoperative biliary drainage (log-rank $\chi 2=4.965, p=0.026$ ) were also related to prognosis (Table 2, Fig. $4 A-G$,).

Table 2 - Univariate analysis of potential predictors for prognosis of HC patients.

\begin{tabular}{|c|c|c|c|c|c|}
\hline Item & & $\mathbf{n}$ & $\begin{array}{l}\text { Median of survival } \\
\text { time (month) }\end{array}$ & $\begin{array}{c}\text { OS } \\
\text { rate(\%) }\end{array}$ & $\mathbf{p}$ \\
\hline \multirow{2}{*}{ Age } & $<60$ years & 97 & 41.1 & $34.0 \%$ & \multirow{2}{*}{0.983} \\
\hline & $\geq 60$ years & 84 & 41.3 & $33.3 \%$ & \\
\hline \multirow{2}{*}{ Sex } & Male & 103 & 41.0 & $34.0 \%$ & \multirow{2}{*}{0.953} \\
\hline & Female & 78 & 41.5 & $33.3 \%$ & \\
\hline \multirow{2}{*}{ Tumor size } & $<2.5 \mathrm{~cm}$ & 114 & 42.4 & $36.0 \%$ & \multirow{2}{*}{0.320} \\
\hline & $\geq 2.5 \mathrm{~cm}$ & 67 & 39.3 & $29.9 \%$ & \\
\hline \multirow{2}{*}{ Differentiation } & High and moderate & 174 & 42.1 & $33.9 \%$ & \multirow{2}{*}{0.060} \\
\hline & Poor & 7 & 20.7 & $28.6 \%$ & \\
\hline \multirow{2}{*}{ Bismuth-Corlette classification } & $|+| I$ & 42 & 48.2 & $45.2 \%$ & \multirow{2}{*}{0.036} \\
\hline & IIIa+IIIb+IV & 139 & 39.1 & $30.2 \%$ & \\
\hline \multirow{2}{*}{ Lymph node metastasis } & Negative & 117 & 45.0 & $37.8 \%$ & \multirow{2}{*}{0.007} \\
\hline & Positive & 64 & 33.9 & $25.8 \%$ & \\
\hline \multirow{2}{*}{ Nerve invasion } & Negative & 109 & 42.3 & $38.5 \%$ & \multirow{2}{*}{0.134} \\
\hline & Positive & 72 & 39.6 & $26.4 \%$ & \\
\hline \multirow{2}{*}{ Portal vein invasion } & Negative & 126 & 42.9 & $38.1 \%$ & \multirow{2}{*}{0.027} \\
\hline & Positive & 55 & 37.4 & $23.6 \%$ & \\
\hline \multirow{2}{*}{ Hepatic artery invasion } & Negative & 142 & 42.5 & $37.3 \%$ & \multirow{2}{*}{0.019} \\
\hline & Positive & 39 & 36.4 & $20.5 \%$ & \\
\hline \multirow{2}{*}{ Liver invasion } & Negative & 132 & 44.0 & $35.6 \%$ & \multirow{2}{*}{0.030} \\
\hline & Positive & 49 & 33.8 & $28.6 \%$ & \\
\hline \multirow{2}{*}{ Incised margin } & Negative(R0) & 151 & 43.5 & $37.1 \%$ & \multirow{2}{*}{0.001} \\
\hline & Positive(R1) & 30 & 29.6 & $16.7 \%$ & \\
\hline \multirow{2}{*}{ Total bilirubin } & $<100 \mathrm{mg} / \mathrm{L}$ & 77 & 40.7 & $35.1 \%$ & \multirow{2}{*}{0.868} \\
\hline & $\geq 100 \mathrm{mg} / \mathrm{L}$ & 104 & 41.6 & $32.7 \%$ & \\
\hline \multirow{2}{*}{ Preoperative biliary drainage } & Undone & 126 & 42.8 & $38.9 \%$ & \multirow{2}{*}{0.026} \\
\hline & Done & 55 & 37.6 & $21.8 \%$ & \\
\hline \multirow{2}{*}{ Postoperative radiotherapy } & Undone & 109 & 40.9 & $33.0 \%$ & \multirow{2}{*}{0.825} \\
\hline & Done & 72 & 41.8 & $34.7 \%$ & \\
\hline \multirow{2}{*}{ MMP-9 } & $<201.93 \mathrm{ng} / \mathrm{mL}$ & 75 & 50.9 & $69.3 \%$ & \multirow{2}{*}{$<0.001$} \\
\hline & $\geq 201.93 \mathrm{ng} / \mathrm{mL}$ & 106 & 34.5 & $8.5 \%$ & \\
\hline
\end{tabular}

Note: OS: overall survival; MMP-9: matrix metalloproteinase-9. 
A

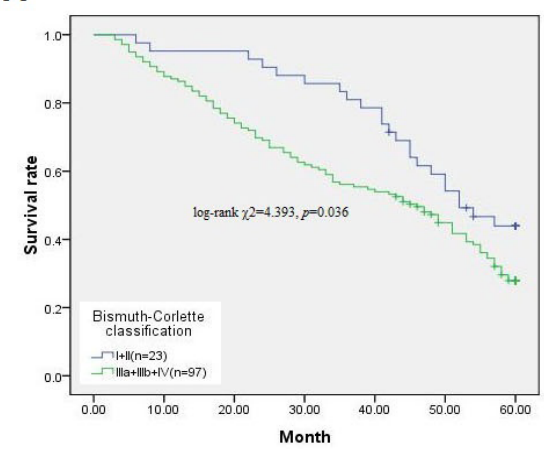

C

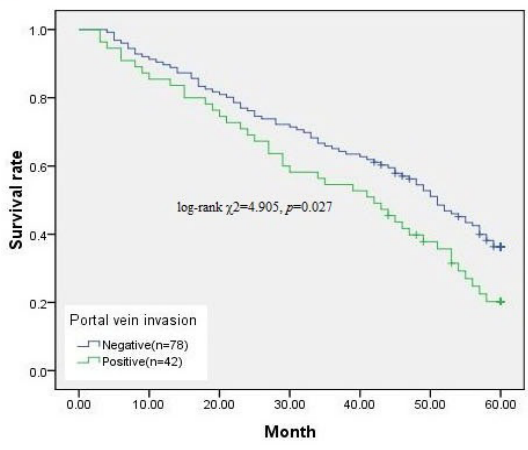

E

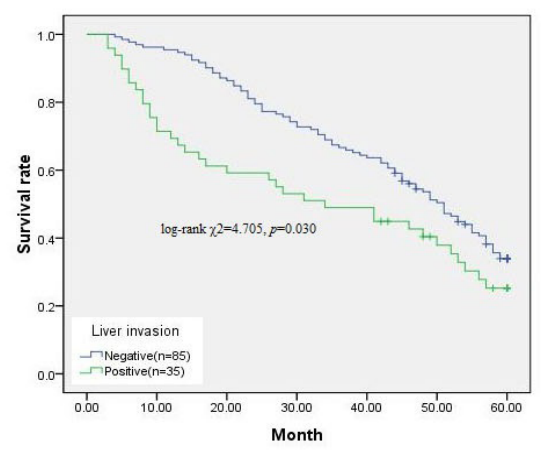

G

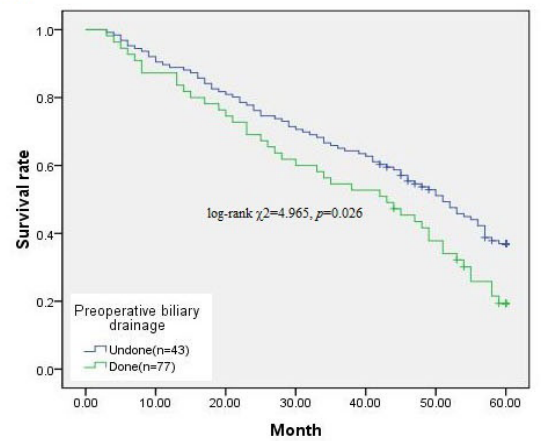

B

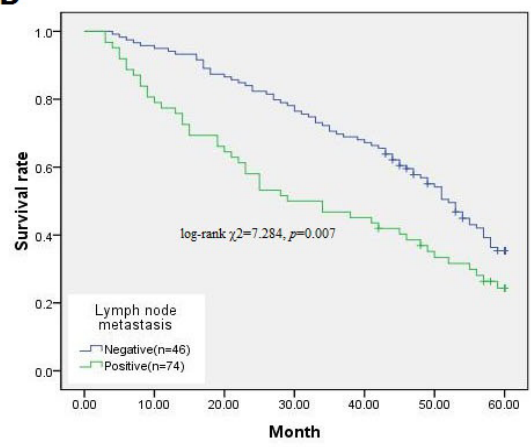

D

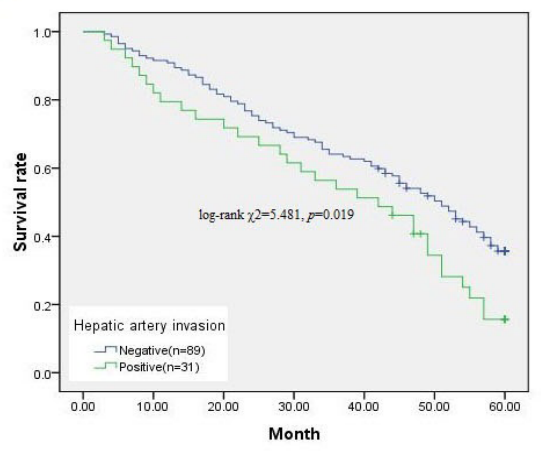

$\mathbf{F}$

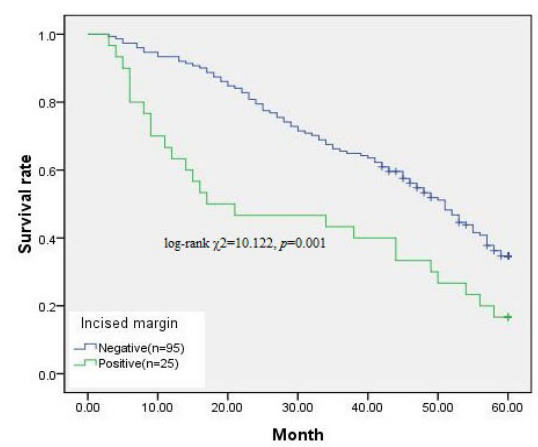

H

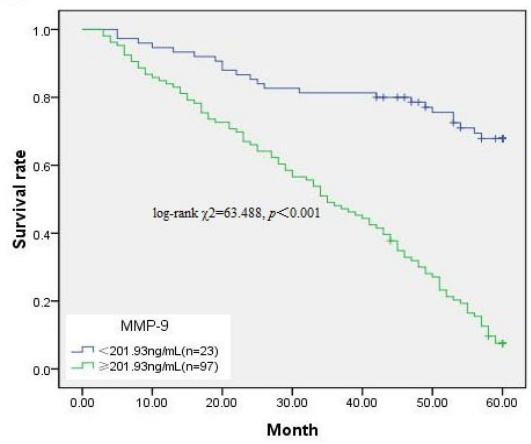

Figure 4 - Relationship between clinical pathological parameters and preoperative serum MMP-9 and postoperative survival of HC patients. A: Bismuth-Corlette classification. B: Lymph node metastasis. C: Portal vein invasion. D: Hepatic artery invasion. E: Liver invasion. F: Incised margin. G: Preoperative biliary drainage. H: Preoperative serum MMP-9. " $n$ " represents the number of deaths. 


\section{Cox regression model}

Next, we included the statistically significant differences in the results of the univariate analysis into the Cox regression model for multivariate analysis. Multivariate analysis confirmed that hepatic artery invasion $(p=0.016)$, liver invasion $(p=0.044)$, incised margin $(p=0.012)$, and MMP-9 $(p<0.001)$ have the potential to independently predicate prognosis in $\mathrm{HC}$ patients (Table 3 ).

Table 3 - Multivariate analysis of potential predictors for prognosis of HC patients.

\begin{tabular}{llll}
\hline Variable & HR & Chi-square & $p$ value \\
\hline Lymph node metastasis & 1.430 & 3.388 & 0.066 \\
Hepatic artery invasion & 1.710 & 5.766 & 0.016 \\
Liver invasion & 1.530 & 4.055 & 0.044 \\
Incised margin & 1.849 & 6.335 & 0.012 \\
Preoperative serum MMP-9 & 5.192 & 61.467 & $<0.001$ \\
\hline
\end{tabular}

Note: MMP-9: matrix metalloproteinase-9; HR: high-risk ratio.

\section{Discussion}

MMPs are one of the most important family of proteolytic enzymes that play an important role in embryonic development, differentiation, tumor angiogenesis, tumor invasion and metastasis ${ }^{9}$. It has been demonstrated that MMPs-mediated degradation of extracellular matrix can lead to invasion and metastasis of tumor cells ${ }^{10,11}$, and abnormal expression of MMPs has prognostic significance in some human malignant tumors ${ }^{12,13}$. Deng et al. ${ }^{14}$ found that MMP-2 protein levels were significantly upregulated in colorectal cancer (CRC), and MMP-2 expression could be novel diagnostic and prognostic markers for CRC patients. Yao et al. ${ }^{15}$ revealed that MMP-2 and MMP-9 expression were both independent factors of prognosis and lymphnode metastasis in patients with early gastric cancer. A meta-analysis also showed a significant poor prognostic effect of MMP-7 in gastric cancer survival ${ }^{16}$. MMP-9 is more well know in the MMPs family, which can degrade type IV collagen in ECM, allowing cancer cells to break through the basement membrane of the primary site ${ }^{17}$. MMP-9 can attenuate the basement membrane of blood vessels and lymphatic vessels, allowing cancer cells to infiltrate directly into the vasculature ${ }^{18}$ to participate in tumor metastasis and invasion ${ }^{19}$. Some scholars have conducted immunohistochemistry to confirm that MMP-9 has a high value in predicting the long-term prognosis of multiple tumors ${ }^{20,21}$. Zhao et al. ${ }^{22}$ used immunohistochemistry to analyze the expression of MMP-9 in tumor tissues of 127 patients with tirple-negative breast cancer, and found that patients with high MMP-9 expression had poor prognosis. Interestingly, the results of some scholars have been relatively novel, which confirms that MMP-9 may be a protective factor in the carcinogenesis and metastasis of tumors. For example, MMP-9 expression is reduced in some head and neck malignancies with local metastasis ${ }^{23}$ and elevated MMP-9 expression may indicate better overall survival in salivary adenocarcinoma ${ }^{24}$. Even more surprising is that in breast cancer ${ }^{25}$ and enteritis-related cancers $^{26}$, MMP-9 expression also indicates a relatively good prognosis. These results 
suggest that MMP-9 can act as both a tumorpromoting factor and a protective factor, and its relationship with disease prognosis may depend on the specific environment of action.

Sun et $a .^{27}$ found that MMP-9 overexpression was observed in tumor tissues of $46.5 \%$ of patients with cholangiocarcinoma of the liver. Their study also found that although MMP-9 overexpression was not associated with patient clinicopathological parameters, overall survival was significantly lower in patients with high MMP-9 expression than in patients with negative or low MMP9 expression, suggesting that the expression of MMP-9 in tissues is of great significance in the evaluation of postoperative prognosis of intrahepatic cholangiocarcinoma. Recent studies have shown that real-time monitoring of non-invasive serum biomarkers targeting prognosis has greater safety and efficacy ${ }^{28}$. MMP-9 levels in the peripheral circulation have also been shown to be associated with metastasis and long-term prognosis in a variety of cancers. Sung et al. ${ }^{29}$ tested the preoperative serum MMP-9 levels in breast cancer patients and found that elevated MMP9 levels were associated with decreased breast cancer survival rate. Lin et $a .^{30}$ also reported that plasma MMP-9 was significantly elevated in thyroid cancer patients with lymph node invasion and distant metastasis, and suggested that regular detection of plasma MMP-9 may help to determine the distant metastasis tendency. However, the relationship between preoperative serum MMP-9 levels and postoperative prognosis in $\mathrm{HC}$ patients has not been reported. Therefore, we focused on serum MMP-9 levels and studied the relationship between preoperative serum MMP-9 levels and postoperative prognosis in HC patients.

We used the ROC curve method to analyze the MMP-9 cut-off point, which has the most prognostic value after $\mathrm{HC}$ radical surgery. The results showed that the area under the ROC curve was the largest ( $A \cup C=0.896)$ when the cut-off point was $201.93 \mathrm{ng} / \mathrm{mL}$, which was the most valuable value. Kaplan-Meier analysis confirmed that the median survival time of HC patients with low serum MMP9 levels was significantly higher than that of patients with high serum MMP-9 levels. These results confirm that preoperative serum MMP-9 can be used as a useful marker to predict postoperative prognosis in $\mathrm{HC}$ patients. Furthermore, we found that preoperative serum MMP-9 levels were associated with Bismuth-Corlette classification and Lymph node metastasis, which may be related to the lower sensitivity of MMP-9 in predicting the prognosis of $\mathrm{HC}$ patients. Our results suggested that MMP-9 may be involved in the progression and metastasis of $\mathrm{HC}$ disease, which may be related to the potential carcinogenesis of MMP-9, but the related mechanism remains to be further explored. We further included statistically significant clinical pathological factors and preoperative serum MMP-9 levels in the multivariate survival analysis model. It was found that high serum MMP-9 levels is an independent risk factor for predicting the prognosis of HC patients. Additionally, Cox analysis showed that risk factors associated with prognosis of patients with $\mathrm{HC}$ include portal vein invasion, hepatic artery invasion, liver invasion, preoperative biliary drainage, etc., which may also affect the sensitivity of MMP-9 by affecting the immune response state of the body.

\section{Conclusions}

As far as we know, this is the only study to evaluate the relationship between preoperative serum MMP-9 levels and postoperative prognosis in $\mathrm{HC}$ patients. In 
this study, we showed that high MMP-9 levels have a high predictive value for postoperative prognosis in patients with $\mathrm{HC}$ undergoing radical resection, and are one of the independent factors influencing postoperative prognosis. Therefore, evaluation of serum MMP-9 levels may be helpful in guiding the prognosis of $\mathrm{HC}$ patients and subsequent treatment options. However, more studies are necessary to confirm our data.

\section{References}

1. Soares KC, Kamel I, Cosgrove DP, Herman JM, Pawlik T M. Hilar cholangiocarcinoma: diagnosis, treatment options, and management. Hepatobiliary Surg Nutr. 2014;3(1):18-34. doi: 10.3978/j.issn.23043881.2014.02.05.

2. Sudan D, DeRoover A, Chinnakotla S, Fox I, Shaw Jr B, McCashland T, Sorrell M, Tempero M, Langnas $A$. Radiochemotherapy and transplantation allow long-term survival for nonresectable hilar cholangiocarcinoma. Am J Transplant. 2002;2(8):774-9. PMID: 12243499.

3. Matsuyama R, Mori R, Ota Y, Homma $\mathrm{Y}$, Kumamoto T, Takeda K, Morioka D, Maegawa J, Endo I. Significance of vascular resection and reconstruction in surgery for hilar cholangiocarcinoma: with special reference to hepatic arterial resection and reconstruction. Ann Surg Oncol. 2016;23(Suppl 4):475-84. doi: 10.1245/ s10434-016-5381-2.

4. Lee SG, Song GW, Hwang S, Ha TY, Moon DB, Jung $\mathrm{DH}$, Kim KH, Ahn CS, Kim MH, Lee SK, Sung KB, Ko GY, Surgical treatment of hilar cholangiocarcinoma in the new era: the Asan experience. J Hepatobiliary Pancreat Sci. 2010;17(4):476-89. doi: 10.1007/ s00534-009-0204-5.

5. Vafadari B, Salamian A, Kaczmarek L. MMP-9 in translation: from molecule to brain physiology, pathology, and therapy. J Neurochem. 2016;139 Suppl 2:91-114. doi: 10.1111/jnc.13415.

6. Dragutinovic VV, Radonjic NV, Petronijevic ND, Tatic SB, Dimitrijevic IB, Radovanovic NS, Krivokapic ZV. Matrix metalloproteinase-2 (MMP-2) and -9 (MMP-9) in preoperative serum as independent prognostic markers in patients with colorectal cancer. Mol Cell Biochem. 2011;355(1-2):173-8. doi: 10.1007/s11010-011-0851-0.

7. Burlaka AP, Ganusevich II, Gafurov MR, Lukin SM, Sidorik EP. Stomach cancer: interconnection between the Redox state, activity of MMP-2, MMP-9 and stage of tumor growth. Cancer Microenviron. 2016;9(1):27-32. doi: 10.1007/s12307-0160182-5.

8. SchveigertD, Cicenas S, Bruzas S, Samalavicius NE, Gudleviciene Z, Didziapetriene J. The value of MMP-9 for breast and non-small cell lung cancer patients' survival. Adv Med Sci. 2013;58(1):73-82. doi: 10.2478/ v10039-012-0066-y.

9. Sun XF, Zhang H. Clinicopathological significance of stromal variables: angiogenesis, lymphangiogenesis, inflammatory infiltration, MMP and $\mathrm{PINCH}$ in colorectal carcinomas. Mol Cancer. 2006;5:43. doi: 10.1186/1476-4598-5-43.

10.Fink K, Boratynski J. The role of metalloproteinases in modification of extracellular matrix in invasivetumorgrowth, metastasis and angiogenesis. Postepy Hig Med Dosw (Online). 2012;66:609-28. doi: 10.5604/17322693.1009705.

11.Santibanez JF, Obradovic H, Kukolj T, Krstic J. Transforming growth factor-beta, matrix metalloproteinases, and urokinase-type plasminogen activator interaction in the cancer epithelial to mesenchymal transition. Dev Dyn. 2018;247(3):382-95. doi: 10.1002/ dvdy.24554.

12.Jensen AS, Vainer B, Bartels A, Brunner $\mathrm{N}$, Sorensen JB. Expression of matrix metalloproteinase 9 (MMP-9) and tissue inhibitor of metalloproteinases 1 (TIMP-1) by colorectal cancer cells and adjacent stroma cells--associations with histopathology and patients outcome. Eur J Cancer. 2010;46(18):3233-42. doi: 10.1016/j.ejca.2010.07.046.

13. Molica S, Vitelli G, Levato D, Giannarelli D, Vacca A, Cuneo A, Cavazzini F, Squillace $R$, Mirabelli R, Digiesi, G. Increased serum levels of matrix metalloproteinase-9 predict clinical outcome of patients with early B-cell chronic lymphocytic leukaemia. Eur J Heamatol. 2003;70(6):373-8. doi: 10.1034/j.1600-0609.2003.00064.x. 
14.Deng J, Chen W, Du Y, Wang W, Zhang G, Tang Y, Qian Z, Xu P, Cao Z, Zhou Y. Synergistic efficacy of Cullin1 and MMP-2 expressions in diagnosis and prognosis of colorectal cancer. Cancer Bio. 2017;19(1):57-64. doi: 10.3233/CBM-160341.

15.Yao $Z$, Yuan $T$, Wang $H$, Yao $S$, Zhao $Y$, Liu $Y$, Jin S, Chu J, Xu Y, Zhou W, Yang $\mathrm{S}$, Liu Y. MMP-2 together with MMP-9 overexpression correlated with lymph node metastasis and poor prognosis in early gastric carcinoma. Tumour Biol. 2017;39(6):1010428317700411. doi: $10.1177 / 1010428317700411$.

16.Soleyman-Jahi $S$, Nedjat $S$, Abdirad A, Hoorshad N, Heidari R, Zendehdel K. Prognostic significance of matrix metalloproteinase-7 in gastric cancer survival: a meta-analysis. Plos one. 2014;10(4):e0122316. doi: 10.1371/journal. pone. 0122316 .

17.Zhang M, Zhu GY, Gao HY, Zhao SP, Xue Y. Expression of tissue levels of matrix metalloproteinases and tissue inhibitors of metalloproteinases in gastric adenocarcinoma. J Surg Oncol. 2011;103(3):243-7. doi: 10.1002/jso.21824.

18.Roy R, Yang J, Moses MA. Matrix metalloproteinases as novel biomarkers and potential therapeutic targets in human cancer. J Clin Oncol. 2009;27(31):5287-97. doi: $10.1200 /$ jco.2009.23.5556.

19.Herszenyi L, Hritz I, Lakatos G, Varga $M Z$, Tulassay $Z$. The behavior of matrix metalloproteinases and their inhibitors in colorectal cancer. Int J Mol Sci. 2012;13(10):13240-63. doi: 10.3390/ ijms131013240.

20.Salem N, Kamal I, Al-Maghrabi J, Abuzenadah A, Peer-Zada AA, Qari Y, Al-Ahwal M, AlQahtani $M$, Buhmeida A. High expression of matrix metalloproteinases: MMP-2 and MMP-9 predicts poor survival outcome in colorectal carcinoma. Future Oncol (London, England). 2016;12(3):323-31. doi: 10.2217/ fon.15.325.

21.Merdad A, Karim S, Schulten HJ, Dallol A, Buhmeida A, Al-Thubaity F, Gari MA, Chaudhary AG, Abuzenadah AM, Al-Qahtani $\mathrm{MH}$. Expression of matrix metalloproteinases (MMPs) in primary human breast cancer: MMP-9 as a potential biomarker for cancer invasion and metastasis. Anticancer Res.
2014;34(3):1355-66. doi: 10.1186/14712407-9-188.

22.Zhao S, Ma W, Zhang $M$, Tang $D$, Shi $Q$, Xu S, Zhang $X$, Liu $Y$, Song $Y$, Liu L, Zhang $Q$. High expression of CD147 and MMP-9 is correlated with poor prognosis of triplenegative breast cancer (TNBC) patients. Med Oncol. 2013;30(1):335. doi: 10.1007/ s12032-012-0335-4.

23.Stokes A, Joutsa J, Ala-Aho R, Pitchers $M$, Pennington CJ, Martin C, Premachandra DJ, Okada Y, Peltonen J, Grenman R, James HÁ, Edwards DR, Kahari VM. Expression profiles and clinical correlations of degradome components in the tumor microenvironment of head and neck squamous cell carcinoma. Clin Cancer Res. 2010;16(7):2022-35. doi: 10.1158/1078-0432.CCR-09-2525.

24.Luukkaa H, Klemi P, Leivo I, Makitie AA, Irish J, Gilbert R, Perez-Ordonez B, Hirsimaki P, Vahlberg T, Kivisaari A, Kahari VM, Grenman R. Expression of matrix metalloproteinase-1, $-7,-9,-13$, Ki-67, and HER-2 in epithelialmyoepithelial salivary gland cancer. Head Neck. 2010;32(8):1019-27. doi: 10.1002/ hed.21277.

25. Bendrik C, Robertson J, Gauldie J, Dabrosin C. Gene transfer of matrix metalloproteinase-9 induces tumor regression of breast cancer in vivo. Cancer Res. 2008;68(9):3405-12. doi: 10.1158/0008-5472.CAN-08-0295.

26.Garg P, Sarma D, Jeppsson S, Patel NR, Gewirtz AT, Merlin D, Sitaraman SV. Matrix metalloproteinase- 9 functions as a tumor suppressor in colitis-associated cancer. Cancer Res. 2010;70(2):792-801. doi: 10.1158/0008-5472.CAN-09-3166.

27.Sun Q, Zhao C, Xia L, He Z, Lu Z, Liu C, Jia $M$, Wang J, Niu J. High expression of matrix metalloproteinase-9 indicates poor prognosis in human hilar cholangiocarcinoma. Int J Clin Exp Pathol. 2014;7(9):6157-64. PMID: 25337264.

28.Kurosaki A, Hasegawa K, Kato T, Abe K, Hanaoka T, Miyara A, O'Shannessy DJ, Somers EB, Yasuda M, Sekino T, Fujiwara K. Serum folate receptor alpha as a biomarker for ovarian cancer: Implications for diagnosis, prognosis and predicting its local tumor expression. Int J Cancer. 2016;138(8):1994-2002. doi: 10.1002/ ijc. 29937.

29.Sung H, Choi JY, Lee AS, Lee KM, Han S, Jeon 
S, Song M, Lee Y, Park SK, Yoo KY, Noh DY, Ahn SH, Kang D. The association between the preoperative serum levels of lipocalin-2 and matrix metalloproteinase-9 (MMP-9) and prognosis of breast cancer. BMC Cancer. 2012;12:193. doi: 10.1186/1471-2407-12193.
30.Lin SY, Wang YY, Sheu WH. Preoperative plasma concentrations of vascular endothelial growth factor and matrix metalloproteinase 9 are associated with stage progression in papillary thyroid cancer. Clin Endocrino. 2003;58(4):513-8. doi: 10.1046/j.1365-2265.2003.01749.x.

\section{Correspondence:}

Chuang Peng

Department of Hepatobiliary Surgery, Hunan

Provincial People's Hospital

61 Jiefang West Road, Changsha 410005

People's Republic of China

Phone: +86 0731-83929139

chuangpeng66@126.com

Received: Dec 19, 2018

Review: Feb 16, 2019

Accepted: Mar 14, 2019

\section{Conflict of interest: none}

Financial source: none

This is an Open Access article distributed under the terms of the Creative Commons Attribution License, which permits unrestricted use, distribution, and reproduction in any medium, provided the original work is properly cited. 